\title{
Philosophiques
}

\section{L’anthropologie politique et le matérialisme décomplexé \\ Benoît Dubreuil, Human Evolution and the Origins of Hierarchies}

\section{Dave Anctil}

Volume 39, numéro 1, printemps 2012

URI : https://id.erudit.org/iderudit/1011618ar

DOI : https://doi.org/10.7202/1011618ar

Aller au sommaire du numéro

Éditeur(s)

Société de philosophie du Québec

ISSN

0316-2923 (imprimé)

1492-1391 (numérique)

Découvrir la revue

Citer ce document

Anctil, D. (2012). L’anthropologie politique et le matérialisme décomplexé /

Benoît Dubreuil, Human Evolution and the Origins of Hierarchies.

Philosophiques, 39(1), 251-258. https://doi.org/10.7202/1011618ar d'utilisation que vous pouvez consulter en ligne.

https://apropos.erudit.org/fr/usagers/politique-dutilisation/ 


\title{
Disputatio \\ L'anthropologie politique et le matérialisme décomplexé
}

\author{
DAVE ANCTIL \\ Collège Jean-de-Brébeuf
}

Human Evolution and the Origins of Hierarchies mobilise une quantité impressionnante de connaissances scientifiques sur l'anthropogenèse des institutions sociopolitiques. Il est rare de voir ainsi réunie, avec compétence et clarté, une somme aussi considérable de données aux registres disciplinaires aussi variés. L'ouvrage témoigne en effet d'une connaissance approfondie des recherches de pointe expliquant l'origine et la formation des hiérarchies sociales, mobilisant nombre de données les plus récentes en archéologie, en neuroscience et en primatologie. Les sciences sociales, l'épistémologie et la philosophie politique ne sont pourtant pas en reste dans l'étude de Benoît Dubreuil. L'auteur discute et emploie certains outils fondamentaux de ces disciplines pour parvenir à son objectif, soit offrir une synthèse originale détaillant le «tournant» évolutionniste de l'humanité archaïque à l'époque du néolithique, ainsi que de nouveaux paramètres explicatifs pour interpréter la diversité des trajectoires sociales de l'humain.

Les prémisses de la démonstration ne manquent certainement pas d'audace pour un philosophe et politologue de formation. Dans son ouvrage, Dubreuil souscrit en effet à une « approche naturaliste » sur l'évolution de la culture et des institutions humaines. Or le naturalisme appliqué à l'anthropologie sociale est généralement mal perçu par les philosophes et les anthropologues culturalistes. Le risque est bien entendu de relancer une querelle inique (la question du "nature ou culture») qui contribue à séparer radicalement les contributions, pourtant complémentaires, des études fondées sur l'observation des données biophysiques, et celles fondées sur l'interprétation sociogénétique et culturelle des institutions sociales. Dubreuil y revient d'ailleurs dans sa conclusion en rappelant que:

[...] the naturalist's contribution to the social sciences is neither to deny that institutions are created through somehow contingent historical processes nor to minimize the astounding diversity of present and past social arrangements, but to explain why regularities still exist in human affairs. Institutions are anchored in expectations, which in turn are always defined contextually, and this suffices to explain why human cultural productions are always unique (p. 229).

À cet égard, j'estime que l'auteur remporte son pari haut la main. Il évite soigneusement toute forme de réductionnisme mal avisé en construisant 
une argumentation nuancée établissant toujours les limites explicatives des mécanismes évoqués. Sur le plan épistémologique, son approche invoque à la fois le fonctionnalisme et le cognitivisme. Elle fait en outre appel aux "mécanismes explicatifs» en s'appuyant sur les travaux de Jon Elster, de Charles Tilly et de bien d'autres. Cette approche, certes risquée pour un sujet d'une telle ampleur, a néanmoins le grand mérite de faire collaborer plusieurs registres explicatifs interdépendants pour clarifier la sélection d'hypothèses très prometteuses pour la recherche anthropologique contemporaine.

Il me semble par ailleurs évident que les sciences humaines ne peuvent plus ignorer la centralité du naturalisme en décriant son prétendu "réductionnisme» (souvent au nom de la sauvegarde de leur crédo académique). Puisque les informations culturelles et sociales sont générées et interprétées par des cerveaux humains, les transmissions culturelles sont nécessairement organisées et modulées par des capacités cognitives génétiquement déterminées. Observer les «régularités» dans les comportements et dans l'interprétation de l'information culturelle est absolument nécessaire pour quiconque veut offrir des explications convaincantes de l'évolution sociale. Les capacités cognitives spécifiques aux hominidés, longuement discutées par Dubreuil, fournissent en effet les trajectoires nécessairement limitées par lesquelles les informations peuvent transiter pour construire le sens culturel et social. La contribution des neurosciences des dernières décennies nous permet déjà de mieux circonscrire ces paramètres de la cognition sociale.

Toute méthodologie sérieuse dans l'étude anthropologique doit donc pouvoir intégrer la cognition humaine dans l'économie générale de son cadre explicatif. Car l'évolution culturelle et sociale dépend tout à la fois de l'information culturelle et des caractéristiques génétiques et neurocognitives. En d'autres mots, les humains sont déterminés par les caractéristiques héritées de notre évolution biologique et culturelle (Distin, 20I I). Comme le soutient Dubreuil tout au long de son ouvrage, l'immense variété culturelle et institutionnelle qui constitue les individus et les groupes humains résulte d'interactions uniques et innombrables entre la diversité des génotypes et la diversité des environnements naturels et sociaux. L'étude et la compréhension de cette diversité dans les trajectoires évolutionnistes offrent des défis complexes et stimulants pour renouveler l'anthropologie de demain.

Cela dit, je ne pourrais prétendre porter un jugement critique - c'està-dire éclairé et compétent - sur l'utilisation des données zoologiques et neuropsychologiques des trois premiers chapitres de l'ouvrage qui dépassent largement ma compétence. En conséquence, ma discussion de l'argument de Benoît Dubreuil se concentrera sur la thèse générale qui est graduellement développée dans les deux derniers chapitres de son ouvrage, et plus particulièrement la question centrale portant sur l'anthropogenèse des hiérarchies sociales et étatiques. Plus généralement, je voudrais questionner certains aspects méthodologiques et épistémologiques de son approche qui a l'ambi- 
tion, à mon avis très noble, de vouloir renouveler les fondements de l'anthropologie politique.

L'origine des inégalités sociales - et en particulier celle de l'État - est une question ancienne - voire une question existentielle - pour laquelle il n'existe encore aujourd'hui aucun consensus anthropologique dans les sciences naturelles ou sociales. C'est la question à laquelle se sont attaqués les grands fondateurs de l'anthropologie politique: Machiavel, Hobbes et Rousseau - tradition vénérable que Dubreuil convoque parfois avec bonheur dans certains passages de son ouvrage. Jusqu'à un certain point, en vertu ou à cause de la quantité mirobolante de données fournies par la recherche évolutionniste contemporaine, il s'agit d'une question à laquelle il est devenu si difficile de répondre qu'elle fut marginalisée à travers l'essor de la spécialisation scientifique au tournant des années I960-I970. Ce n'est donc pas la moindre contribution de Dubreuil que de remettre cet enjeu fondamental sur la table. Bien entendu, les travaux évolutionnistes de deuxième génération (neoevolutionism) ont permis de découvrir une multitude de régularités anthropologiques entre les cultures qui ont confirmé la valeur heuristique du cadre explicatif évolutionniste. C'est au chapitre IV que l'auteur introduit et discute ces explications néo-évolutionnistes qui ont contribué à mieux distinguer les formes d'organisations sociales hiérarchiques en typologies diverses. Au chapitre V, il discute ensuite la diversité des approches théoriques qui ont questionné l'origine des États pour ensuite mettre à profit cette discussion afin d'étudier certains paramètres dans les processus de démocratisation des sociétés.

La méthode choisie par l'auteur est remarquable à plus d'un égard. Il ne tente pas d'invalider les approches généalogiques de l'État pour en consacrer une au mépris d'autres contributions. Au contraire: l'auteur choisit plutôt de capitaliser, dans une approche syncrétique aussi rigoureuse qu'audacieuse, sur ce qu'il y a de vrai et d'utile dans chaque cadre théorique ${ }^{1}$. Il convient de souligner cet effort - pourtant condamné à l'avance à l'imperfection des raccourcis et aux liens causaux douteux - , car c'est la seule manière de procéder pour quiconque espère offrir une synthèse probante sur un sujet aussi vaste et complexe.

Rappelons maintenant la ligne directrice de l'argument de l'ouvrage. Comment expliquer l'origine des inégalités sociales, dans la mesure où les sociétés humaines "primitives » paraissaient plutôt égalitaires? Le préjugé anthropologique - depuis au moins Rousseau selon Dubreuil - est de présupposer une passion originaire pour l'égalité. Le postulat de l'égalité naturelle a logiquement poussé des générations de philosophes et d'anthropologues à interpréter l'évolution vers la civilisation comme un processus transforma-

1. Je n'aborderai pas en détail le volet épistémologique de la congruence explicative des sciences naturelles et sociales puisque je partage avec l'auteur les grands traits de l' 'ecumenical stance» développée par le philosophe Philip Pettit (I993). 
teur et corrupteur de la "nature égalitariste» de l'humain. Dubreuil opte plutôt pour une autre hypothèse de départ: la "passion pour la punition" est plus fondamentale pour comprendre à la fois les normes égalitaires et l'origine des hiérarchies. C'est par un travail innovateur faisant largement place à la cognition que l'auteur va étayer les fondements anthropologiques des hiérarchies sociales et l'émergence de l'État. Observons maintenant les grands traits de cette démonstration.

Le rôle cognitif des émotions (comme l'envie, la colère, la honte ou l'indignation) étudié au premier chapitre est central à la compréhension des dimensions affectives de la socialité, en particulier le conformisme aux normes et la punition de la déviance. Tout le chapitre II vise à nous convaincre, à la lumière des données paléoanthropologiques, que la psychologie de l'humain moderne est à ce titre unique et le distingue de ses cousins hominidés ou primates qui ne possèdent pas les mêmes dispositions à s'organiser socialement. C'est au chapitre III que Dubreuil développe ces dispositions cognitives uniques d'homo sapiens qui sont apparues au cours de son évolution neurobiologique. L'auteur accorde une place moins centrale au langage qu'à certaines fonctions cognitives permettant à l'humain de construire et de transmettre des représentations symboliques complexes, inaccessibles aux autres hominidés, qui ont permis l'élargissement démographique et la stratification hiérarchique des sociétés. Comment? Ce n'est uniquement qu'au chapitre IV qu'est finalement développé le «lien fonctionnaliste» permettant d'expliquer le passage des petites sociétés égalitaires aux grandes sociétés inégalitaires.

L'argument des quatre premiers chapitres est le suivant: étant donné l'architecture émotionnelle et cognitive unique dont est doté homo sapiens, il est bien moins coûteux pour les individus de sanctionner des étrangers que des congénères puisque, pour simplifier, les premiers individus ne ressentant pas aussi fortement les émotions associées à la sanction que les seconds, il est alors nécessaire de développer d'autres moyens de les sanctionner. L'hypothèse explicative globale paraît être la suivante: plus une société est nombreuse, plus elle doit «investir» dans la punition de la déviance. C'est pourquoi, selon Dubreuil, les mécanismes cognitifs ayant conduit à l'élaboration d'institutions permettant la "délégation des sanctions" à des tiers sont si importants pour comprendre l'anthropogenèse de l'État. D'abord, ces dispositions humaines en sont une condition nécessaire: sans les dispositions neuropsychologiques adaptées pour effectuer ces opérations complexes, homo sapiens ne vivrait pas dans des sociétés organisées aussi nombreuses et socialement complexes. Ces dispositions cognitives sont également une condition (partiellement) suffisante puisque ces institutions ont été graduellement développées pour répondre aux coûts sans cesse croissants associés au maintien organisé et prévisible du conformisme social.

Les étapes de cette longue démonstration, parfois très technique, sont les piliers de la thèse de l'auteur sur l'anthropogenèse politique, déployée 
dans les deux derniers chapitres. Les fondements naturels de l'évolution des institutions de pouvoir, notamment les dispositifs émotionnels et cognitifs organisant le rôle de la sanction dans la coopération sociale, servent en somme à expliquer la tendance vers l'État. L'argument de la «délégation de la sanction " redéployé au chapitre $\mathrm{V}$ est l'outil principal expliquant le passage graduel des sociétés primitives décentralisées aux États régulateurs centralisés. Pourquoi les humains, à l'encontre de leur tendance à vouloir punir directement les déviants, en viennent-ils à confier l'autorité à une instance centrale de décision, capable de déléguer le pouvoir à des instances secondaires pour sanctionner la déviance aux normes sociales, et de l'autoriser à le faire? Dubreuil répond: parce que, le lien social étant essentiellement fondé sur les émotions partagées et la capacité de sanctionner la déviance aux normes, les dispositifs hiérarchiques de l'État sont plus efficaces et moins coûteux pour organiser la punition conformiste dans les grandes sociétés où le lien social est, par nécessité, plus ténu.

L'explication quasi-fonctionnaliste de Dubreuil a le grand mérite d'attirer notre attention sur des paramètres échappant aux explications traditionnelles qui ne font pas (ou peu) de cas des spécificités cognitives humaines. Toutefois, les facteurs cognitifs ne sont pas, à mon avis, les plus importants pour expliquer la genèse de l'État; en tout cas, ils ne méritent pas, à la lumière de l'état actuel de nos connaissances neuropsychologiques, une telle centralité. D'autres aspects plus importants, tels les facteurs matériels structurant les rivalités sociales, permettent d'élargir l'explication à une causalité plus complexe et plus crédible.

En effet, le peu de place accordé à l'étude des structures élémentaires de la conflictualité humaine me semble être la principale faiblesse de l'argument global de Human Evolution. Les aspects non cognitifs de la conflictualité des groupes humains - les rapports matériels de pouvoir et de concurrence sont peu étudiés. Il me semble pourtant que cette conflictualité a occasionné la part la plus importante de la division inégalitaire du travail social. En remplaçant la «passion pour l'égalité » par une «passion pour la punition conformiste» comme prémisse de départ de l'anthropologie politique, Dubreuil se retrouve en quelque sorte à «naturaliser les hiérarchies». De sorte qu'il évite soigneusement de prendre position sur certains enjeux philosophiques et méthodologiques auxquels j'aimerais maintenant le convier.

L'existence des inégalités considérables dans les sociétés complexes paraît contre-intuitive à quiconque étudie l'anthropologie. Ce problème de la «servitude volontaire» est souligné avec brio par l'auteur qui demande avec raison pourquoi les humains acceptent aussi facilement les inégalités même lorsqu'ils n’y sont pas forcés (p. I 83). La réponse de Dubreuil au chapitre IV repose en bonne partie sur une analyse fine des stratégies déployées par les individus ambitieux pour construire et asseoir leur autorité sur leurs semblables, soit l'intégration sociale par réseaux ou par incorporation (p. I77-I 8 I). 
Cette discussion des hiérarchies pose problème dans la mesure où, dans l'argument présenté, tout se passe comme si l'apparition des hiérarchies répondait toujours à une même demande d'adaptation fonctionnaliste. L'auteur précise certes que les adaptions locales furent d'une très grande diversité. Toutefois, la plupart des changements socio-institutionnels connus par les préhistoriens et les historiens sont apparus à travers des conflits d'autorité ou en présence d'une menace extérieure hostile. Ma première critique est donc que l'argument fonctionnaliste de Dubreuil ne nous permet pas de comprendre suffisamment pourquoi les conflits intragroupes et intergroupes apparaissent, puisque la genèse des hiérarchies sociales est essentiellement liée à la réduction des coûts de la sanction. En quoi l'argument de Human Evolution peut-il nous aider à comprendre les structures élémentaires de la conflictualité sociale qui président aux épisodes de grandes transformations sociales?

Ma propre hypothèse est que, pour étudier les structures élémentaires de la conflictualité humaine, et en particulier l'histoire des rivalités violentes qui ont dessiné les contours de l'évolution des hiérarchies sociales, il convient de s'inscrire dans un cadre explicatif pleinement matérialiste. Un tel matérialisme doit en outre intégrer les déterminismes associés aux milieux environnementaux et, en particulier, aux changements matériels écologiques qui ont structuré de manière importante les trajectoires sociales humaines (ce que l'auteur reconnaît partiellement en 2.5.I au moment d'étudier la grande «flexibilité écologique» d'homo sapiens). Par exemple, les sociétés eurasiennes ont bénéficié d'avantages comparatifs associés à l'environnement, ce qui leur a permis de construire des sociétés hiérarchiques et conquérantes. Ces sociétés ont joui d'une très grande variété naturelle de plantes et d'animaux domesticables. Les avantages en productivité agricole de l'Eurasie expliquent de nombreux aspects des trajectoires de leurs civilisations et, en bonne partie, la naissance archaïque des premières formes d'État dans ces aires géographiques. Dans l'évolution de ces groupes humains, la coordination des accès aux ressources matérielles a joué un rôle prépondérant. Or il n'est pas évident de percevoir comment la contribution de Dubreuil est susceptible de nous aider à mieux comprendre, par exemple, la «tendance à l'oligarchie» chez homo sapiens. Depuis Aristote, véritable fondateur de l'anthropologie, cette tendance à l'accumulation et à l'avantage comparatif a pourtant été inscrite dans la nature de la conflictualité historique humaine.

À mon avis, s'inscrire dans une approche naturaliste comme celle de Dubreuil implique en somme de souscrire à une conception matérialiste élargie de l'évolution humaine. Un tel «matérialisme décomplexé » constitue l'orientation philosophique et méthodologique à laquelle - implicitement ou explicitement - adhèrent la plupart des chercheurs dans les sciences naturelles. Mais cette orientation est aujourd'hui bien moins commune chez les philosophes de formation (p. ex. Searle, 2004). Car soutenir que la compréhension des phénomènes biophysiques doit être au cœur de l'ensemble 
des mécanismes causaux de l'évolution humaine va à l'encontre du lourd héritage structuraliste des sciences humaines. C'est aussi pourquoi les sciences humaines sont marquées par le poids d'une tradition donnant la place prépondérante au langage et aux constructions idéales. Ce faisant, les chercheurs ont aisément tendance à minimiser non seulement les facteurs cognitifs étudiés par Dubreuil, mais aussi d'autres facteurs biophysiques et environnementaux sur les sociétés. Étant donné l'influence importante des sciences humaines sur l'éducation et les échanges d'informations entre citoyens, cela incite peut-être nos sociétés à surestimer leur capacité à s'adapter artificiellement (culturellement et technologiquement) aux changements matériels des conditions d'existence (comme les changements climatiques ou la destruction de la biodiversité).

C'est pourquoi les étiquettes de "déterminisme biologique» ou de «réductionnisme environnemental» ne devraient jamais faire craindre aux philosophes de s'inscrire dans une perspective matérialiste pour faire contrepoids à certaines tendances antiscientifiques des sciences humaines. Toutefois, en insistant autant sur les facteurs cognitifs, Dubreuil choisit peut-être un peu arbitrairement une forme indirecte du matérialisme, centré sur la neuropsychologie, à l'exclusion d'autres facteurs biophysiques environnementaux qui paraitront plus importants aux historiens évolutionnistes des sociétés humaines.

Selon mon point de vue, l'anthropologie politique doit graduellement devenir une discipline pleinement biohistorique: elle doit convoquer réciproquement la généalogie des impacts biophysiques sur l'histoire humaine avec l'histoire de l'impact de l'activité humaine sur la nature (Russell, 20I I). À mon avis, toute approche naturaliste devrait donc être plus équilibrée en faisant appel à d'autres éléments de l'histoire naturelle que les grands traits neurocognitifs des hominidés. Je pense notamment aux impacts de la géographie et des systèmes environnementaux, comme le climat et les microorganismes, sur les changements dans les conditions matérielles d'existence des sociétés humaines. Ces facteurs matériels peuvent nous aider à comprendre une grande quantité de contraintes exercées sur les trajectoires historiques des sociétés humaines (Diamond, 2000 et 2006).

En terminant, j'estime que Human Evolution and the Origins of Hierarchies est très certainement l'une des contributions majeures à l'anthropologie politique de la dernière décennie. Je salue Benoît Dubreuil pour cet effort titanesque qui nous permet de jeter un regard nouveau sur l'origine des hiérarchies humaines.

\section{Références}

Diamond, Jared. I997. De l'inégalité parmi les sociétés, trad. P.-E. Dauzat, Paris, Gallimard, 2000.

- 2005. Effondrement. Comment les sociétés décident de leur disparition ou de leur survie, trad. A. Botz et J.-L. Fidel, Paris, Gallimard, 2006. 
Distin, Kate. Cultural Evolution, Cambridge, Cambridge Univ. Press, 20 I I.

Pettit, Philip. The Common Mind: An Essay on Psychology, Society, and Politics, New York, Oxford Univ. Press, I993.

Russell, Edmund. Evolutionary History. Uniting History and Biology to Understand Life on Earth, Cambridge, Cambridge Univ. Press, 20 I I.

Searle, John. R. Freedom \& Neurobiology. Reflections on Free Will, Language, and Political Power, New York, Columbia Univ. Press, 2004. 